\title{
Sclerotherapy with Leuprolide and Cefoperazone Sulbactam in the Management of Ovarian Endometriomas under Ultrasound Guidance: A Novel Approach
}

\author{
Yazhini Selvaraj \\ Ponni Hospital and Fertility Research Centre, Madurai, India \\ Email: Yazhinisevaraj1956@gmail.com
}

How to cite this paper: Selvaraj, Y. (2022) Sclerotherapy with Leuprolide and Cefoperazone Sulbactam in the Management of Ovarian Endometriomas under Ultrasound Guidance: A Novel Approach. Advances in Sexual Medicine, 12, 34-46.

https://doi.org/10.4236/asm.2022.121004

Received: October 27, 2021

Accepted: January 2, 2022

Published: January 5, 2022

Copyright ( 2022 by author(s) and Scientific Research Publishing Inc. This work is licensed under the Creative Commons Attribution International License (CC BY 4.0).

http://creativecommons.org/licenses/by/4.0/ (c) (i) Open Access

\begin{abstract}
Endometriosis is an estrogen dependent gynecological disorder, which can occur throughout the reproductive age of women, causing chronic pelvic pain, substantial morbidity, and impaired fertility. It is clinically defined as the presence of ectopic endometrium resulting in sustained inflammatory reaction. In India, about 29,000,000 women suffer from endometriosis. Severe pelvic pain and recurrent endometriomas were observed even after surgery. We have conducted aspirations totally in 110 patients in which most of the patients were suffering from Stage III \& Stage IV of endometriosis except for 7 patients for whom we have done as primary therapy for endometriomas. Among 110 patients, 4 patients didn't turn up for follow up after first aspiration. This pilot study conducted with 110 patients in Ponni Hospital and Fertility Research Centre, Madurai aimed to evaluate pregnancy outcome in 51 infertile patients, and to alleviate pelvic pain, to restore a healthy sexual life and to reduce the recurrence rate of Ovarian Endometriomas (OE) in 59 patients, who did not seek fertility, After using the newer sclerosing agent Leuprolide with Cefoperazone Sulbactam (LCS) under ultrasound guidance. This prospective pilot study resulted in a highly significant achievement in pregnancy where 41 patients conceived out of 51 patients (80.39\%) only 6 are in ongoing treatment. Those who didn't seek fertility were 59 of which 43 have completed family 13 have one child and 3 unmarried girls. $96.61 \%$ were relieved of pain and recurrence and restored healthy sexual life, 2 are in ongoing treatment. We have not encountered any adverse effects during this treatment.
\end{abstract}

\section{Keywords}

Ovarian Endometriomas (OE), Infertility (IF), Sclerosing Agent (SA), 


\section{Introduction}

Endometriosis is found predominantly in women of reproductive age group. More than 200 million women worldwide and 29 million women in India suffer from endometriosis. 50\% of infertile women suffer from endometriosis. It is predominantly found in women during their childbearing age 15 to 49 years. About $30 \%-50 \%$ of infertile women are suffering with endometriosis [1], Ovarian Endometriomas (OE) are prevalent in $40 \%$ to $82.5 \%$ of women, suffering from endometriosis [2]. It is described as the appearance of endometrial tissue and stroma outside of the uterus. It is an estrogen dependent gynecological disorder which results in severe pelvic pain, dysmenorrhea, dyspareunia, dyschezia and may cause infertility [3]. The possible causes for infertility are Ovulatory dysfunction like abnormal folliculogenesis, Anovulation, Luteal phase defect, Luteinization of un-rupture follicle syndrome, Immunological alterations like decreased sperm survival, altered immunity, Peritoneal factors like intraperitoneal inflammation, local production of prostaglandins, cytokins, interference with implantation associated with endometrial dysfunction, mechanical factors like anatomical distortion of tubes, altered tubal motility, interference with ovum pickup, peritubal adhesions, Interference with coital function, due to dyspareunia and sperm inactivation due to phagocytosis by macrophages. The different stages of endometriosis are stage I (Minimal), stage II (Mild), stage III (Moderate), stage IV (Severe). This can be diagnosed by using Ultrasound for severe endometriosis, and for primary or early endometriosis definitive diagnosis, is possible only with laparoscopy. Endometriosis may be symptomatic or asymptomatic associated with pelvic pain and/or infertility, can be diagnosed through physical examination findings such as fixed retroverted uterus, an adnexal mass, uterosacral ligament nodularity, thickening or tenderness. There are a lot of medical therapies postulated to treat endometriosis, the principle of medical therapy is to induce amenorrhea and to create a hypo-estrogenic environment as endometriosis is an estrogen dependent disease. By creating a hypo-estrogenic environment the growths of endometriotic lesions are temporarily arrested, either we should create a pseudo pregnancy state or pseudo menopausal state in order to promote temporary regression of the endometriotic lesions. The adverse effects of this therapy may be intolerability and all symptoms can recur after stopping the treatment. Fertility cannot be achieved since ovulation is inhibited during this therapy. We have to choose the medical therapy according to the effectiveness, tolerability, cost of drug, physician experience and expected patient compliance.

\section{Risk Factor and Treatment for Endometriosis}

The initial treatment option for pelvic pain is pelvic pain management. Proges- 
tins are the most effective medication for pain management and deep infiltrating endometriosis, with or without dyspareunia and dysmenorrhea. Ovarian Endometriomas are cystic lesions, which contain thick dark brown colored fluid, and are generally referred to as chocolate cysts [4]. It can even lead to the development of cancer rarely and hypoestrogenic state with anovulation [5]. This usually affects the patients, who are anxious to conceive. The other option to treat endometriosis is through surgical procedure, which includes laparoscopic cystectomy or fulguration which is very useful but the recurrence rate of endometriosis is relatively high, causing a negative effect in ovarian reserve due to devastation of healthy ovarian tissue adjacent to the cyst wall. It may also result in complications such as rectovaginal fistula [6].

Endometriosis is a chronic inflammatory disease. Due to the chronic intraperitoneal inflammation, it can reduce the fertility by increasing pro-inflammatory cytokines. It may interfere with oocyte-sperm interactions, embryo development and implantation [7]. The risks associated with endometriosis are nulliparity, early menarche, late menopause, short menstrual cycles (less than 27 days), heavy menstrual bleeding, Mullerian anomalies, exposure to diethylstilbestrol in utero and endometriosis is first degree relatives. The main clinical expressions of the disease are chronic pelvic pain and infertility. $71 \%$ to $87 \%$ of the women suffered from the chronic pelvic pain [8]. It may have a negative effect in the sexual wellbeing, quality of life and efficacy [9].

To preserve the ovarian reserve, nonsurgical management of $\mathrm{OE}$, including endometriomas aspiration, or sclerotherapy, has been supported. It is widely used to reduce the recurrence of $\mathrm{OE}$. This sclerotherapy includes aspiration of $\mathrm{OE}$ and instilling the cyst cavity with sclerosing agent like Ethanol, Injection Tetracycline (which is banned in India) and Methotrexate. Although these sclerosingagents are cost-effective, it has limitations like abdominal pain, post-operative fever, alcohol intoxication and intracystic abscess formation [10]. The newer sclerosing agents are Leuprolide with Cefoperazone Salbactam (LCS), Interferons or Interleukins (1), Recombinant Interleukin (2), Metronidazole.

\section{Sclerotheraphy}

The endometriosis management is done using 1) the conventional method: expectant management, medical therapy and surgery 2) Sclerotherapy with Ethanol, Tetracycline, or Methotrexate as sclerosing agents. Repeated surgeries are not only a financial burden but also damage the ovarian reserve badly, whereas medical management of endometriomas are really costly and it has to be continued for a longer spell of time during which fertility management couldn't be undertaken. In 1939, Sclerotherapy was introduced by McCausland in US. Synthetic sclerosants were invented in 1940s and 1950s. In India, it is often considered as the treatment of small varicose vein. This sclerotherapy entails injecting sclerosants directly into vein. It causes scar in vein forcing the blood to reroute through healthier vein; the crumbled vein is reabsorbed into the tissue and 
eventually fades. [11]. Sclerotherapy was originally used to treat tuberculous pneumonitis and is currently used by medical oncologist to treat maligant pleural effusion [12]. It is also a first line treatment for Lympahtic Malformations (LMs), using a sclerosing agent like sodium tertradecyl sulphate (STS) [13]. It is a novel cheap out-patient procedure (OP). This modality is now used in treating endometriomas. It requires injecting a sclerosing agent such as Ethanol, Methotrexate and Tetracycline into the cyst cavity after drainage.

\section{Why Sclerotheraphy?}

In the year 2016, a patient with severe endometriomas, who had already undergone three surgeries and was threatened by her husband and in-laws to be divorced if she doesn't beget a child within a year. She and her parents were sobbing in my cabin, the plight of this patient triggered my empathy towards her and made me search the internet for an alternative therapy for endometriomas, which could quickly relieve her symptoms and restore her fertility at a shorter time. That's the time when I came across the treatment called sclerotherapy which was practiced in US, Chinese Taipei, Japan, Canada, Israel and some European countries and regions.

Cohen et al., in his retrospective study, have stated that the sclerosing agent ethanol and tetracycline have some common complications like higher rate of recurrence, abdominal pain, and sclerosing agent like methotrexate causes postoperative fever.

To overcome the limitations of these sclerosing agents, we came up with novel approach, of sclerotherapy using Leuprolide with Cefoperazone Salbactam (LCS). This is used to instill the cyst cavity, after completing the aspiration under Trans-vaginal sonography guidance. In this present study, we achieved a minimal recurrence rate of $\mathrm{OE}$ and good pregnancy outcome in infertile patients using the newer sclerosing agent Leuprolide with Cefoperazone Salbactam (LCS) to treat endometriomas under ultrasound guidance. Figure 1 shows the diagram of endometriosis.

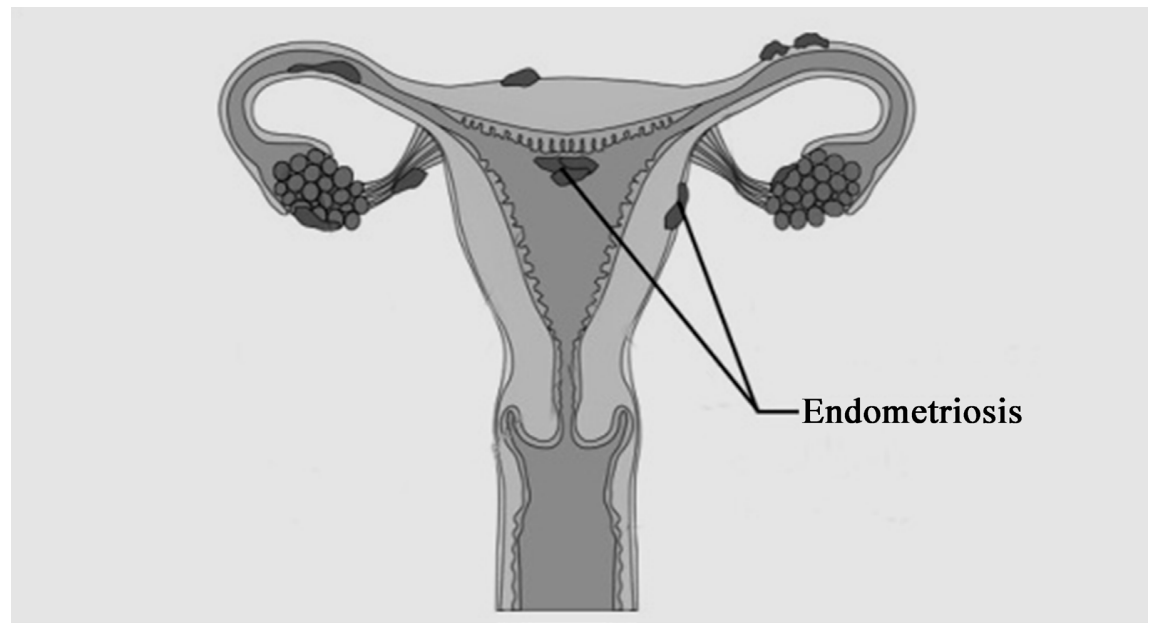

Figure 1. Endometriosis. 


\section{Materials and Methods}

\subsection{Inclusion and Exclusion Criteria}

From April 2016 till date, Scleortherapy was done using a newer sclerosing agent namely Leuprolide with Cefoperazone Salbactam (LCS) at Ponni Hospital and Fertility Research Center, Madurai for treating endometriomas. This prospective pilot study included 110 patients, in which 51 were infertile women and 59 who did not seek fertility treatment. The inclusion criteria for this study were: $\mathrm{Pa}$ tients who had recurrent endometriomas, severe pelvic pain with or without infertility, endometriotic cyst with size more than $5 \mathrm{~cm}$, those suffering from stage III \& IV endometriosis and those who had previous surgical interventions. The exclusion criteria for this study were patients who had no associated pathologies like fibroids, tuberculosis, and any other gynecological pathology. Age is not a factor. We collected signed informed consent from patients who were included in this study after proper explanation (counseled) of the treatment limitations and risk. Table 1 shows the inclusion and exclusion criteria of our patients.

\subsection{Demographic Characteristics}

Out of all the 110 patients, 106 patients were in follow up, 51patients were infertile and 59 patients did not seek fertility treatment.7 patients had primary aspirations. The rest were in stage III and IV of endometriosis. Among the 59 patients who did not seek fertility treatment, 43 patients had completed family, 13 patients had only one child and 3 unmarried girls. The average age of patient was 31 \pm 6 years and average size of endometriosis was $5-15 \mathrm{~cm} .4$ patients didn't turn up for follow up. Table 2 shows the socio-demographic study of our patients.

\subsection{Administration Protocol}

Under aseptic precautions and under antibiotic coverage, with transvaginal

Table 1. Inclusion and Exclusion criteria.

\begin{tabular}{ll}
\hline Inclusion Criteria & Exclusion Criteria \\
\hline Infertile women & Pelvic Inflammatory Disease \\
Women in reproductive age & Fibroid \\
Repeated/Recurrent Surgeries & Chronic Cervicitis/Vaginitis (after treating) \\
Previous Laparoscopy Cystectomy (endometriotic) & Adnexal Pathology (Solid Ovarian Mass) \\
Yet to complete family & Tuberculosis Pelvis \\
Recurrent endometriomas & Endometriomas of $<5 \mathrm{~cm}$ \\
Primary/Stage III/Stage IV & Hydrosalpinx \\
Cyst size $>5$ cm & Any malignancy in reproductive organs \\
Low AMH & \\
Low AFC & \\
Without other gynecological disorder & \\
\hline
\end{tabular}


Table 2. Socio-Demographic Study of our 110 patients.

\begin{tabular}{|c|c|c|}
\hline Variables & & $\mathrm{N}=110$ \\
\hline \multicolumn{3}{|l|}{ Age (yrs) } \\
\hline $20-30$ & & $45(41 \%)$ \\
\hline $30-40$ & & $22(20 \%)$ \\
\hline$>40$ & & $43(39 \%)$ \\
\hline \multicolumn{3}{|l|}{ Education } \\
\hline Degree & & $74(67 \%)$ \\
\hline Schooling & & $36(33 \%)$ \\
\hline \multicolumn{3}{|l|}{ Religion } \\
\hline Hindu & & $97(88 \%)$ \\
\hline Other Religion & & $13(12 \%)$ \\
\hline \multicolumn{3}{|l|}{ Marital Status } \\
\hline Married > $10 \mathrm{yrs}$ & & $59(54 \%)$ \\
\hline Married $\leq 10$ yrs. & & $48(43.63 \%)$ \\
\hline Unmarried & & $3(2.72 \%)$ \\
\hline \multicolumn{3}{|l|}{ Ethnicity } \\
\hline Indian & & $109(99 \%)$ \\
\hline Non-Residential Indian & & $1(1 \%)$ \\
\hline \multicolumn{3}{|l|}{ Employment } \\
\hline Working & & $37(34 \%)$ \\
\hline Homemaker & & $73(66 \%)$ \\
\hline \multicolumn{3}{|l|}{ Income } \\
\hline Low Class & & $32(29 \%)$ \\
\hline Middle Class & & $47(43 \%)$ \\
\hline Upper Middle Class & & $31(28 \%)$ \\
\hline \multicolumn{3}{|l|}{ Patient Category } \\
\hline \multirow{4}{*}{$\begin{array}{l}\text { Patients Not Seeking } \\
\text { Fertility Treatment }\end{array}$} & Family Completed & $43(39 \%)$ \\
\hline & Yet to complete family & $13(11.8 \%)$ \\
\hline & Unmarried & $3(3 \%)$ \\
\hline & Divorcee & $1(1 \%)$ \\
\hline \multirow{2}{*}{$\begin{array}{l}\text { Patients Seeking Fertility } \\
\text { Treatment }\end{array}$} & Primary Infertility & $43(39 \%)$ \\
\hline & Secondary Infertility & $8(7.2 \%)$ \\
\hline
\end{tabular}

ultrasound guidance, a 17 gauge $/ 37 \mathrm{~cm}$, single-lumen aspiration needle was inserted into the endometriomas. Figure 2 shows the single and double lumen needle and craft suction apparatus. 
The cyst contents were aspirated, and cyst cavity was washed with normal saline until aspirated fluid was clear, averagely size of the cyst ranged from 5 - 15 $\mathrm{cms}$, and the average amount of fluid was $25-30 \mathrm{ml}$. The largest endometriotic cyst aspirated had $800 \mathrm{ml}$ of endometriotic fluid. In case of thick and old endometriomas, aspiration of the endometriotic fluid was achieved using 16 gauge $/ 35 \mathrm{~cm}$ Double lumen aspiration needle. Once the aspiration and saline wash was completed, Leuprolide with Cefoperazone Salbactam (LCS) were instilled into the dead space without removing the needle, dose of the sclerosing agents depends according to the size of the cyst.

This entire procedure was conducted as outpatient procedure without anesthesia and only in some apprehensive patients; they were treated under either IV sedation or mild anesthesia. An ultrasound evaluation was performed 5 days to 6 weeks later to assess the efficacy of the treatment. Figure 3 shows the cyst aspiration with the help of 17-gauge needle, reduced cyst, and Instillation of Sclerosing agent.

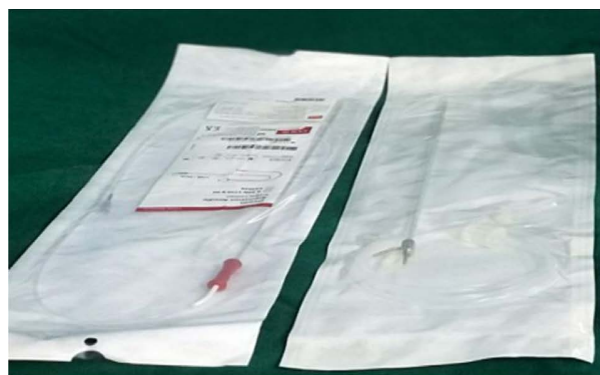

(a)

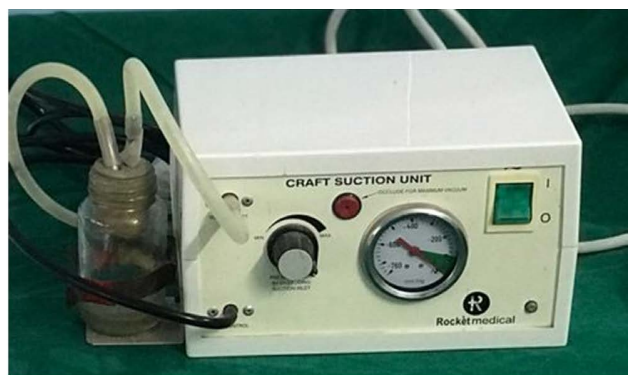

(b)

Figure 2. Ovum Aspiration Needle (single and double) and craft suction. (a) Single and Double Lumen Needle; (b) Craft Suction Apparatus.

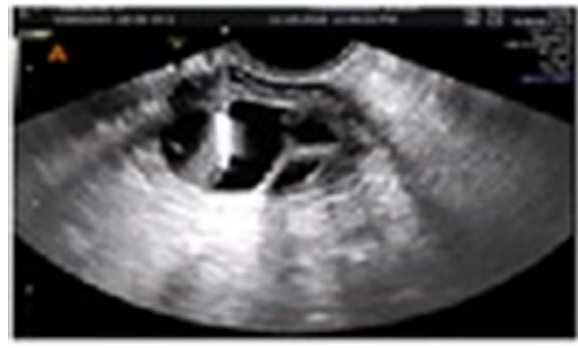

(a)

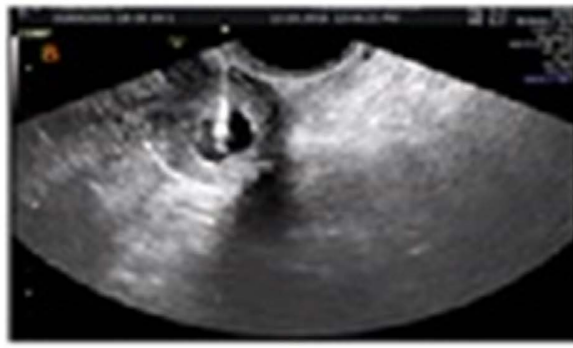

(b)

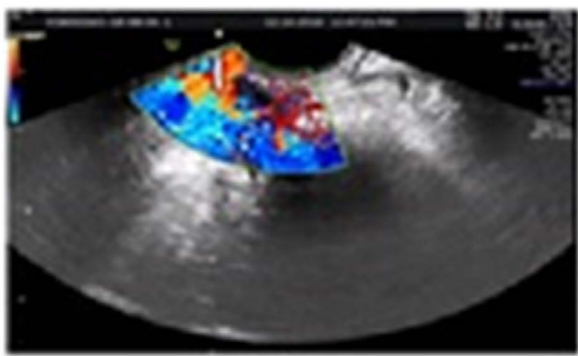

(c)

Figure 3. (a) Cyst aspiration with 17-gauge needle; (b) Reduced Cyst; (c) Instilling the Sclerosing Agent. 


\section{Results}

Among all the 110 patients who underwent sclerotherapy with LCS in our fertility center between April 2016 till date, 51 had infertility problems, and 59 patients did not seek fertility treatment. Among these 110 patients, 106 (96.36\%) were observed with complete resolution at follow-up examination. 4 patients didn't turn up for follow-up after their first aspiration. Among the 51 infertile patients, only $6(11.76 \%)$ patients suffered with recurrence of endometriosis and $1(1.36 \%)$ patient underwent surgery after the sclerotherapy treatment due to repeated recurrences.

Among the 51 patients, 41 of them conceived and delivered. Out of 41 patients 35 (85.36\%) were conceived through IVF and 5 (12.19\%) patients conceived through IUI. 1 (2.43\%) patient had natural conception, 6 patients are in ongoing treatment and 4 patients didn't turn up for follow up. And among the 59 women not seeking fertility, 57 had complete pain score reduction and recurrence rate. Only 2 patients are in going treatment. The pregnancy outcomes among 51 infertile women and particulars about the 59 women not seeking fertility were shown in Table 3. Those 6 patients who are in ongoing treatment among the infertile patients are now being treated with LCS and Metronidazole, and the other 2 patients among the59 women not seeking fertility are being treated with LCS and Methotrexate.

\section{Discussion}

Endometriosis is an estrogen dependent gynecological disorder. Sindan N. et al.

Table 3. Result.

Total no. of Infertile Patients (51)

Live birth achieved

$41(80.39 \%)$

No. of patients conceived through IUI

$5(12.19 \%)$

No. of patients conceived through IVF

$35(85.36 \%)$

No. of patients who conceived naturally

$1(2.43 \%)$

Singleton

$38(92.68 \%)$

Twins

$3(7.31 \%)$

Repeat Aspiration

$4(9.75 \%)$

Single Aspiration

37 (90.24\%)

Laparoscopy after Sclerotherapy

$1(1.96 \%)$

Ongoing treatment

$6(11.76 \%)$ LCS + Metronidazole

Total no. of patients not seeking fertility (59)

Recurrence rate reduction

Pain Score Reduction

Restored Healthy Sexual Life

Ongoing Treatment

$2(3.38 \%)$ with LCS + Methotrexate 
About $30 \%$ to $50 \%$ of the infertile patients are suffering from recurrence of endometriosis and associated with pelvic adhesions, occlusions of the tubal Ostia and decreased folliculogenesis. It is an ever-challenging disease; there must be an individualization of treatment according to the severity of disease and the need for fertility.

First line treatment option for endometriosis is pelvic pain management. Conventionally used medications are NSAIDs (Non-steroidal anti-inflammatory drug), oral contraceptive pills, Gonadotrophin-releasing hormone (GnRH) agonists and aromatase inhibitors [14]. Dopamine Agonist (cabergoline) in the dose of $0.5 \mathrm{mg}$ given inhibits neuro angiogenesis. Dienogest commonly used for long term treatment of endometriosis, $2 \mathrm{mg}$ of dienogest daily effectively diminishes the painful symptoms of endometriosis, alleviates endometriotic lesions, and ameliorates indices of quality of life. But pregnancy can't be achieved with dienogest medication. It is an expensive drug for developing and under developed countries because it ought to be continued for long time (12 - 24 months) [15]. Kolanska $\mathrm{K}$ et al. state that recently, selective progesterone receptor modulators, anti-angiogenic factors and immune modulators are added as a new therapy for endometriosis. The success rate of these medications relatively depends on the age, infertility duration, symptoms severity and stage of disease. The second line treatment option for endometriosis is surgery. The laparoscopic cystectomy with Sharp excision, bipolar diathermy and ablation by $\mathrm{CO}_{2}$-laser techniques have been followed as the gold standard treatment for the OE [16]. The excision of the endometriomas favor pregnancy outcome. However it is unfavorably associated with recurrence of the endometrioma. Following surgery, the ovarian reserve may be reduced as determined by a drop in AMH. Wacharachawana et al. stated that $22.6 \%$ patients suffered from recurrence of the endometriomas after laparoscopic management and pregnancy could only be achieved after a year and a half. Parazzini et al. [17] reported 2-year recurrence rates as14.3\% for stage III and IV endometriosis. Vignali et al. [18] demonstrated that the recurrence rate and pain reduction score in 3 years was $20.9 \%$ and that of 5 -year recurrence rate was $43.5 \%$. Exacoustos et al. [19] expressed that $76 \%$ of patients had pain related symptoms with recurrence of cysts, which were in more than $(>) 10 \mathrm{~cm}$ of endometriomas. Worthington et al. stated that to overcome this recurrence, patients need to take prolong medication for hormone suppression and this indirectly affects the fertility.

Ethanol sclerotherapy (EST) is the most commonly used therapy for treating endometriomas. Laura et al. [20] used $96 \%$ of ethanol to fill cyst cavity which was remained in cavity for 10 mins and was re-aspirated completely. The volume of ethanol instillation ranged from $50 \%$ to $100 \%$ of the initial volume of cyst aspirated.

Sclerotherapy using ethanol caused peritoneal irritation which leads to severe abdominal pain and hence it must be drained as soon as possible and Amparo Garcia et al. also states that this kind of sclerotherapy is not suitable for patients who have malignancy [21]. The main advantage of sclerotherapy is to avoid ag- 
gressive treatment in young woman but the unusual complications caused when the needle is slipped into the vaginal wall, adnexa, or vaginal canal especially when using ethanol produces intense burning due to the chemical reaction with the vaginal mucosa [22]. Wang et.al used 95\% ethanol and $2 \%$ lidocaine to do sclerotherapy and observed adverse effects [23]. Yazbek et al. used 80\% of pure ethanol as sclerosing agent, which was instilled into the cyst, and was left in the cyst for $10 \mathrm{~min}$. This treatment was mainly suggested for infertile patients who wanted to undergo an IVF treatment and not for those who were trying to conceive naturally [24]. Agostini et al. used in-situ methotrexate, which had less toxicity but observed a recurrence rate of $50 \%$ up to one year follow up [25].

The main advantage of sclerotherapy in our studies was that the treatment for infertility can be resumed in the same month or the very next month after aspiration. The other advantages of sclerotherapy were minimal Invasion, cheap and cost-effective, simple OP procedure, with less ovarian manipulation, low recurrence rate, prevents the drop of $\mathrm{AMH}$, resume fertility quickly with a good pregnancy and live birth rate, and avoid GA complications. So far, we have not encountered any adverse effects during this treatment.

Our study is the first novel approach to use LCS as a sclerosing agent for treating endometriomas which were left permanently in situ. It is a GnRH (Gonadotropin releasing Hormone) analog. The mechanism involved in sclerotherapy of Ovarian Endometrioma though not fully understood, it seems that epithelial cells lining the cyst wall play an essential role in bringing adequate contact between the sclerosing agent and the cyst wall which activates coagulation cascade and release of lot of mediators of inflammation causing fibrosis of epithelial lining cells leading to cyst obliteration.

Figure 4 shows the Pretreatment (cyst before the treatment), cyst aspiration and post-treatment using the sclerosing agent LCS. The second mechanism may be this might enhance the cytotoxic activity of macrophages and natural killer cells to suppress the endometrial activity.

Endometriosis an ever-challenging chronic costly disease requires long term multidisciplinary approach. It affects all age groups, from adolescent women to peri-menopausal women. Individualization of treatment is a must according to severity of disease, tolerability of patient and desire for fertility. The standard treatment for symptomatic and endometriomas larger than $5 \mathrm{~cm}$ is laparoscopic surgery.

Dienogest the wonder molecule is preferred mostly, for patient compliance and quicker return of fertility.

This newer sclerosing agent Leuprolide with Cefoperazone Salbactam (LCS) did not cause any abscess, no abdominal pain and no fever. Pregnancy outcome was achieved to $80.39 \%$ in which only six had recurrent endometriomas, and only one patient had to undergo laparoscopic surgery who conceived naturally after surgery. For the 59 patients who did not seek fertility treatment, had good pain score reduction, and had improved sexual health, and only $3 \%$ recurrence 


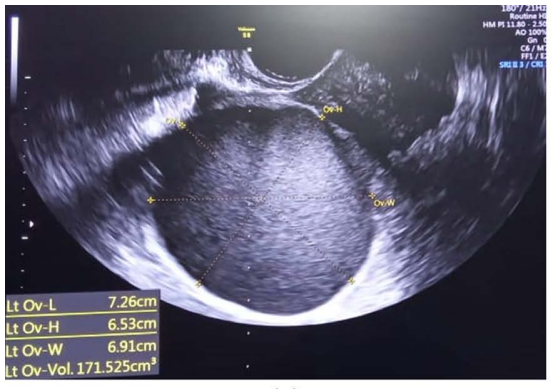

(a)

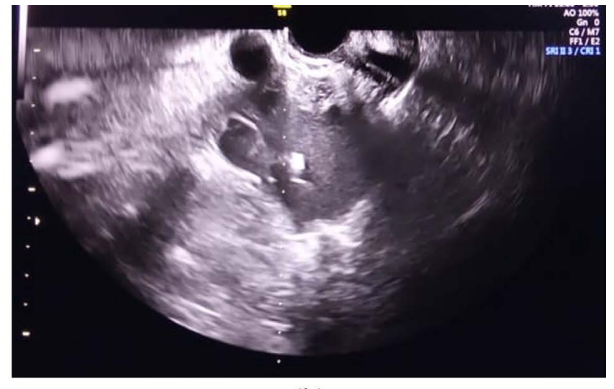

(b)

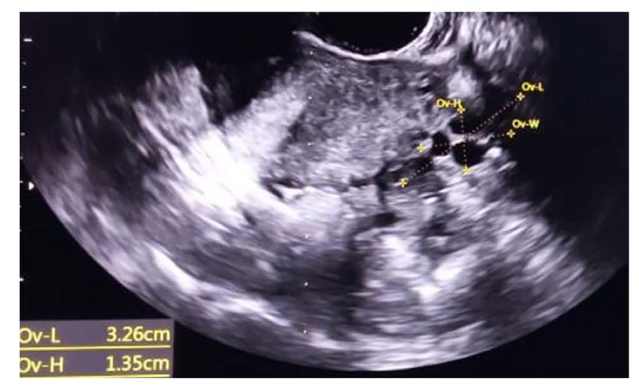

(c)

Figure 4. (a) Pre-Treatment (Right endometriotic cyst); (b) Cyst Aspiration; (c) PostTreatment (Right Ovary with sclerosing agent).

rate noted in our studies. Our patients were followed up to 2 - 6 years, and we achieved $97 \%$ recurrence rate reduction and $80.39 \%$ pregnancy outcome. In this study we further used Leuprolide with Cefoperazone Salbactam (LCS) as sclerosing agent and for the six patients with recurrence we used Metronidazole along with LCS (seeking fertility) and LCS with methotrexate for those not seeking fertility (2 patients).

By using these newer sclerosing agents we could prevent repeated surgeries, achieve good fertility, and restore healthy sexual life. It is a boon to the frustrated endometriotic patients. Sclerotherapy using new sclerosing agents is a Newer Frontier in the management of recurrent endometriomas.

\section{Conflicts of Interest}

The author declares no conflicts of interest regarding the publication of this paper.

\section{References}

[1] Sindan, N., Bhandari, A., Sindan, N., Kc, R., Xia, E. and Lin, Y. (2021) Clinical Factors Influencing the Pregnancy Outcome after Laparoscopic Treatment in Endometriosis-Associated Infertility Patients: A Retrospective Study. American Journal of Translational Research, 13, 2399-2409.

[2] Alborzi, S., Askary, E., Keramati, P., Moradi Alamdarloo, S., Poordast, T., Ashraf, M.A., et al. (2021) Assisted Reproductive Technique Outcomes in Patients with Endometrioma Undergoing Scelerotheraphy vs Laparoscopic Cystectomy: Prospective Cross-Sectional Study. Reproductive Medicine and Biology, 20, 313-320. https://doi.org/10.1002/rmb2.12386

[3] Burney, R.O. and Giudice, L.C. (2012) Pathogenesis and Pathophysiology of Endo- 
metriosis. Fertility and Sterility, 98, 511-519.

https://doi.org/10.1016/j.fertnstert.2012.06.029

[4] Hoyle, A.T. and Puckett, Y. (2021) Endometrioma. StatPearls.

[5] Visconti, F. and Di Carlo, C. (2021) The Etonogestrel Contraceptive Implant as a Therapy for Endometriosis. In: Genazzani, A.R., Nisolle, M., Petraglia, F. and Taylor R.N., Eds., Endometriosis Pathogenesis, Clinical Impact and Management, Springer, Cham, 65-71. https://doi.org/10.1007/978-3-030-57866-4_7

[6] Birbarah, C., Tebache, L., Brichant, G. and Nisolle, M. (2021) What Is the Place of Surgery of Deep Endometriosis in Infertile and Pelvic Pain Patients? In: Genazzani, A.R., Nisolle, M., Petraglia, F. and Taylor, R.N., Eds., Endometriosis Pathogenesis, Clinical Impact and Management, Springer, Cham, 83-95.

https://doi.org/10.1007/978-3-030-57866-4_9

[7] Kolanska, K., Alijotas-Reig, J., Cohen, J., Cheloufi, M., Selleret, L., d'Argent, E., et al. (2021) Endometriosis with Infertility: A Comprehensive Review on the Role of Immune Deregulation and Immunomodulation Therapy. American Journal of Reproductive Immunology, 85, Article ID: e13384. https://doi.org/10.1111/aji.13384

[8] Bloski, T. and Pierson, R. (2008) Endometriosis and Chronic Pelvic Pain: Unraveling the Mystery Behind This Complex Condition. Nursing for Women's Health, 12, 382-395. https://doi.org/10.1111/j.1751-486X.2008.00362.x

[9] Kuznetsov, L., Dworzynski, K., Davies, M. and Overton, C (2017) Diagnosis and Management of endometriosis: Summary of NICE Guidance. BMJ, 358, Article No. j3935. https://doi.org/10.1136/bmj.j3935

[10] Cohen, A., Almog, B. and Tulandi, T. (2017) Scelerotheraphy in the Management of Ovarian Endometrioma: Systematic Review and Meta-Analysis. Fertility and Sterility, 108, 117-124.e5. https://doi.org/10.1016/j.fertnstert.2017.05.015

[11] Worthington-Kirsch, R.L. (2005) Injection Scelerotheraphy. Seminars in Interventional Radiology, 22, 209-217. https://doi.org/10.1055/s-2005-921954

[12] Patz Jr., E.F., McAdams, H.P., Erasmus, J.J., Goodman, P.C., Culhane, D.K., Gilkeson, R.C. and Herndon, J. (1998) Scelerotheraphy for Malignant Pleural Effusions: A Prospective Randomized Trial of Bleomycin vs Doxycycline with Small-Bore Catheter Drainage. Chest, 113, 1305-11. https://doi.org/10.1378/chest.113.5.1305

[13] Barnacle, A.M., Theodorou, M., Maling, S.J. and Abou-Rayyah, Y. (2016) Sclerotherapy Treatment of Orbital Lymphatic Malformations: A Large Single-Centre Experience. British Journal of Ophthalmology, 100, 204-208.

https://doi.org/10.1136/bjophthalmol-2015-306657

[14] Rafique, S. and Decherney, A.H. (2017) Medical Management of Endometriosis. Clinical Obstetrics and Gynecology, 60, 485-496.

https://doi.org/10.1097/GRF.0000000000000292

[15] Schindler, A.E. (2011) Dienogest in Long-Term Treatment of Endometriosis. International Journal of Women's Health, 3, 175-184. https://doi.org/10.2147/IJWH.S5633

[16] Wacharachawana, S., Phaliwong, P., Prommas, S., Smanchat, B., Bhamarapravatana, K. and Suwannarurk, K. (2021) Recurrence Rate and Risk Factors for the Recurrence of Ovarian Endometriosis after Laparoscopic Ovarian Cystectomy. BioMed Research International, 2021, Article ID: 6679641. https://doi.org/10.1155/2021/6679641

[17] Parazzini, F., Bertulessi, C., Pasini, A., Rosati, M., Di Stefano, F., Shonauer, S., et al. (2005) Determinants of Short Term Recurrence Rate of Endometriosis. European Journal of Obstetrics \& Gynecology and Reproductive Biology, 121, 216-219. 
https://doi.org/10.1016/j.ejogrb.2004.11.033

[18] Vignali, M., Bianchi, S., Candiani, M., Spadaccini, G., Oggioni, G. and Busacca, M. (2005) Surgical Treatment of Deep Endometriosis and Risk of Recurrence. Journal of Minimally Invasive Gynecology, 12, 508-513.

https://doi.org/10.1016/j.jmig.2005.06.016

[19] Exacoustos, C., Zupi, E., Amadio, A., Amoroso, C., Szabolcs, B., Romanini, M.E., et al. (2006) Recurrence of Endometriomas after Laparoscopic Removal: Sonographic and Clinical Follow-up and Indication for Second Surgery. Journal of Minimally Invasive Gynecology, 13, 281-288. https://doi.org/10.1016/j.jmig.2006.03.002

[20] Miquel, L., Preaubert, L., Gnisci, A., Resseguier, N., Pivano, A., Perrin, J., et al. (2020) Endometrioma Ethanol Scelerotheraphy Could Increase IVF Live Birth Rate in Women with Moderate-Severe Endometriosis. PLOS ONE, 15, e0239846.

https://doi.org/10.1371/journal.pone.0239846

[21] García-Tejedor, A., Castellarnau, M., Ponce, J., Fernández, M.E. and Burdio, F. (2015) Ethanol Scelerotheraphy of Ovarian Endometrioma: A Safe and Effective Minimal Invasive Procedure. Preliminary Results. European Journal of Obstetrics \& Gynecology and Reproductive Biology, 187, 25-29.

https://doi.org/10.1016/j.ejogrb.2015.02.004

[22] Gatta, G., Parlato, V., Di Grezia, G., Porto, A., Cappabianca, S., Grassi, R. and Rotondo, A. (2010) Ultrasound-Guided Aspiration and Ethanol Sclerotherapy for Treating Endometrial Cysts. La radiologia medica, 115, 1330-1339. https://doi.org/10.1007/s11547-010-0586-0

[23] Wang, S.M., Cai, H.Q., Dong, X.Q., Fan, Q.L., Wang, L.L., Shao, X.H. and Zhang, L. W. (2015) Correlation between Ovarian Chocolate Cyst and Serum Carbohydrate Antigen 125 Level and the Effect of Ultrasound-Guided Interventional Sclerotherapy on Serum Carbohydrate Antigen 125 Level. Journal of Obstetrics and Gynaecology Research, 41, 92-98. https://doi.org/10.1111/jog.12491

[24] Yazbeck, C., Madelenat, P., Ayel, J.P., Jacquesson, L., Bontoux, L.M., Solal, P. and Hazout, A. (2009) Ethanol Sclerotherapy: A Treatment Option for Ovarian Endometriomas before Ovarian Stimulation. Reproductive Biomedicine Online, 19, 121-125. https://doi.org/10.1016/S1472-6483(10)60055-7

[25] Agostini, A., De Lapparent, T., Collette, E., Capelle, M., Cravello, L. and Blanc, B. (2007) In Situ Methotrexate Injection for Treatment of Recurrent Endometriotic Cysts. European Journal of Obstetrics \& Gynecology and Reproductive Biology, 130, 129-131. https://doi.org/10.1016/j.ejogrb.2006.01.015 